\title{
COMPARISON OF BEAM-BASED ALIGNMENT ALGORITHMS FOR THE ILC *
}

\author{
J. C. Smith, L. Gibbons, R. Patterson, D. Rubin, D. Sagan, Cornell University, Ithaca, NY, USA \\ P. Tenenbaum, SLAC, Palo Alto, CA, USA
}

\section{Abstract}

The main linac of the International Linear Collider (ILC) requires more sophisticated alignment techniques than those provided by survey alone. Various BeamBased Alignment (BBA) algorithms have been proposed to achieve the desired low emittance preservation. Dispersion Free Steering, Ballistic Alignment and the Kubo method are compared. Alignment algorithms are also tested in the presence of an Earth-like stray field.

\section{INTRODUCTION}

The International linear Collider (ILC) main linac lattice used in this analysis is based on the TESLA TDR [1] with 23.4 MV/m gradient RF cavities. Crucial to maintaining high luminosity in excess of $10^{34} \mathrm{~cm}^{-2} \mathrm{~s}^{-1}$ is the preservation of the very low vertical emittance $\left(\gamma \epsilon \sim 2 \times 10^{-8} \mathrm{~m}\right)$ from the extraction of the damping rings through to the interaction point. Table 1 gives the uncorrelated misalignments used in the following analysis. Simulations show that the alignment precision necessary to maintain the small emittance far exceeds these installation survey tolerances.

In the current design, magnetic shielding does not cover the entire length of the cryomodules but only the RF cavities. The machine is therefore susceptible to stray fields. Various beam-based alignment algorithms have been proposed to steer the beam to minimize emittance dilution due to misalignments. Here we include the effect of stray DC fields. An analysis has been carried out comparing the effectiveness of three alignment methods in the presence of an Earth-like field applied over the entire length of the main linac. The strength, declination and inclination of the Earth's magnetic field varies around the globe and since the location of the ILC has not yet been chosen we arbitrarily

* Work supported by the U.S. National Science Foundation and the U.S. Department of Energy

\begin{tabular}{|l|c|c|}
\hline Error & Tolerance & With Respect To... \\
\hline Quad Offset & $300 \mu \mathrm{m}$ & Cryostat \\
\hline Quad tilt & $300 \mu \mathrm{rad}$ & Cryostat \\
\hline BPM Offset & $300 \mu \mathrm{m}$ & Cryostat \\
\hline BPM Resolution & $10 \mu \mathrm{m}$ & True Orbit \\
\hline RF Cavity Offset & $300 \mu \mathrm{m}$ & Cryostat \\
\hline RF Cavity Pitch & $200 \mu \mathrm{rad}$ & Cryostat \\
\hline Cryostat Offset & $200 \mu \mathrm{m}$ & Survey Line \\
\hline Cryostat Pitch & $20 \mu \mathrm{rad}$ & Survey Line \\
\hline
\end{tabular}

Table 1: RMS misalignments of the ILC main linac. chose Earth's field in Ithaca NY. The field at ground level is given in Table 2. The orientation of the linac was chosen to be East-West as this orientation will result in the greatest effect on the vertical orbit. This field is strong enough to bend the beam by as much as a few millimeters near the beginning of the linac where the beam energy is only tens of GeV. Simulations were performed in TESLAv, a customized version of the TAO simulation environment [2]. Tao uses the Bmad relativistic charged-particle dynamics library which has been found to agree well in tracking simulations with other codes used in Linear Collider development [3]. A beam of 250 particles was used in the tracking simulations and wakefields are from the TESLA TDR [1]. Initial vertical emittance was set to $20 \mathrm{~nm}$.

\section{BEAM-BASED ALIGNMENT ALGORITHMS}

\section{Ballistic Alignment}

The Ballistic Alignment (BA) algorithm implemented here is similar to that found in [4]. This method first establishes a reference line by turning off all guidefield components within a section of the linac allowing the beam to take a ballistic path and then recording the BPM readings. Assuming there are no stray fields, these BPM readings will define a straight line. Once the optics are restored, the beam is re-steered to this ballistic trajectory. Ideally, one large region would be used to establish a straight reference line through the linac, but it is unlikely that, given the survey tolerances, a straight line will clear all of the apertures over the full $14.3 \mathrm{~km}$ length of the linac. The linac is therefore divided into bins of 7 FODO cells each. The ends of the bins are treated as pivot points where the last BPM in a bin is zeroed before the ballistic orbit is measured.

For 100 seeds, with the misalignments in Table 1 applied, the average final vertical emittance after BA was found to be $25 \mathrm{~nm}$. This is assuming that there are no stray fields, either external to the cryomodule or due to residual fields in the magnets. These results are in agreement with previous results [4].

\begin{tabular}{|l|c|c|}
\hline Field Component & Strength & Unit \\
\hline Magnitude & 54.3 & microTesla \\
\hline Declination & -12.2 & Degrees West \\
\hline Inclination & 69.5 & Degrees Down \\
\hline Linac Orientation & 90.0 & Degrees East \\
\hline
\end{tabular}

Table 2: Earth's magnetic field in Ithaca NY. 


\section{Kubo Method}

Kiyoshi Kubo recently developed another method [5] here-in referred to as the Kubo method. The vast majority of emittance growth is due to dispersion from misaligned quadrupoles. This source can be removed if the kick on each steering magnet exactly cancels the quadrupole kick. The computed kick from the quadrupole is

$$
\theta=k_{1} L_{\text {quad }} Y_{\text {bpm }},
$$

where $k_{1}$ is the quadrupole strength, $L_{\text {quad }}$ is the quadrupole length and $Y_{b p m}$ is the BPM reading. The steering magnet at the quadrupole is set to cancel the quadrupole kick. Crucial to this correction is knowledge of the BPM to quadrupole offset. We assume that quadrupole shunting has been performed and that the rms BPM to quadrupole offset is 30 microns.

For the implementation of the Kubo method used in this paper, the kick, $\theta$, on each steering magnet is set to

$$
\theta=w \theta_{b p m}+k_{1} L_{\text {quad }} Y_{b p m},
$$

where $\theta_{b p m}$ is the kick that would be placed on the steering magnet to zero the orbit at the next downstream BPM and $w$ is the weighting factor for driving the beam to the BPM center. The kick in equation 1 does not constrain the orbit and it may drive it to large amplitudes. The correction in equation 2 keeps the orbit small and the optimum weight was found to be $w=0.01$.

Note that the Kubo method implemented here is different than that originally used in [5]. Here, the correction is applied linearly from the beginning of the lattice to the end whereas [5] used an optimizer to minimize a merit function. Additionally, a full set of design misalignments are applied here, including quadrupole tilts and RF cavity pitches.

Given misalignments similar to those used in [5], the average final emittance for 100 seeds is $28 \mathrm{~nm}$ compared to $34 \mathrm{~nm}$ in the previous analysis. If the full set of misalignments in Table 1 are used, the average final emittance for 100 seeds is $49 \mathrm{~nm}$.

\section{Dispersion Free Steering}

Dispersion Free Steering (DFS) measures and then adjusts the trajectory to minimize the dispersion. The DFS algorithm implemented here is based upon [6]. The beam is steered to zero the BPMs and then the linac is divided into regions of 20 FODO cells where each region overlaps its neighbor by 10 cells. Two orbits are taken for each region. One with the design energy, the other with the energy reduced by $20 \%$ or $18 \mathrm{GeV}$, whichever is less. A LevenbergMarquardt optimizer is then used to find the optimum corrector settings to minimize the orbit and the on- off-energy orbit change where the latter is weighted 100 times greater than the former.

Due to an insufficient number of RF cavities upstream of the first three quadrupoles, DF Steering cannot be used for the low energy end of the linac. Therefore, the first

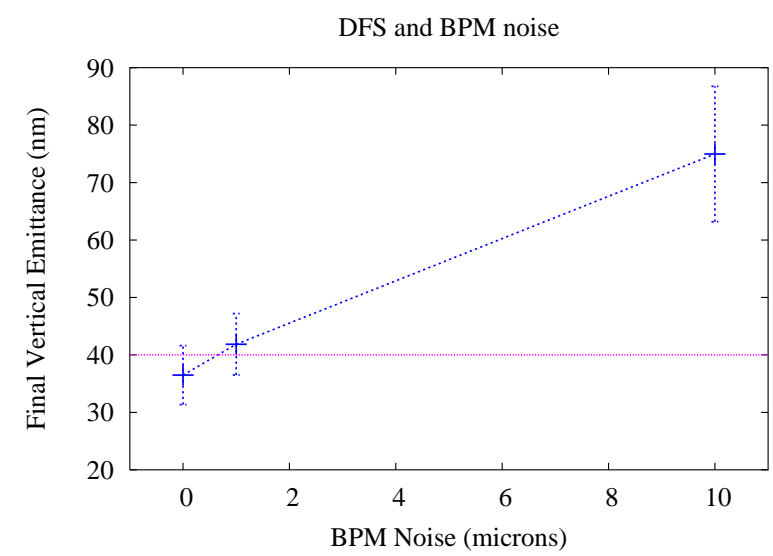

Figure 1: Emittance dilution after DFS for different BPM resolutions.

three quadrupoles and BPMs are assumed to be perfectly aligned. The first DFS region starts right after this third quadrupole. The optimizer runs through 5 LevenbergMarquardt iterations before the next region is steered. Because the incoming orbit into each region will change when the RF cavities are turned off, the incoming orbit must be re-steered to the previous BPM readings before entering the region. 3 BPMs upstream of each region are used to perform this re-steering.

For 100 seeds, with the misalignments in Table 1 applied, the average final vertical emittance after DFS is $92 \mathrm{~nm}$. This is an average emittance dilution of $360 \%$ which is considerably more than the $142 \%$ found in [6]. One significant difference between the current analysis and that of [6] is here quadrupole tilts are being included. However, the main source of discrepancy appears to be due to BPM noise. This is illustrated in Figure 1 which gives the average emittance dilution for different BPM resolutions. Here, quadrupole tilts are not being included and the weight was varied to reflect the change in BPM resolution. With no BPM noise, the results agree with the previous studies $[4,6,7]$. The algorithm implemented here is slightly different and the source of the discrepancy is being investigated. This algorithm uses a different optimizer and resteers the incoming off-energy orbit before each region is DF steered whereas in other algorithms, this re-steering is performed at the same time as the DF steering by including a few BPMs upstream of each DFS region in the optimization.

\section{BBA IN THE PRESENCE OF AN EARTH-LIKE FIELD}

The misalignments in table 1 were applied to the lattice and then an Earth-like dipole field was applied over all unshielded components. Each of the three beam-based alignments was then tested. To compare the sensitivity to the strength of a stray field from another source, the Earth field was varied from 0 to it's full strength of $54.3 \mu \mathrm{T}$. Results 


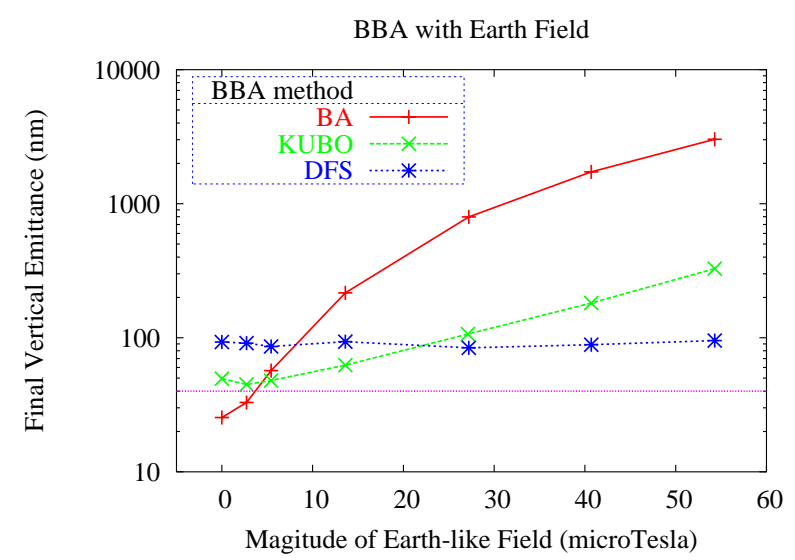

Figure 2: Sensitivity of three BBA algorithms to an Earthlike field of varying strength.

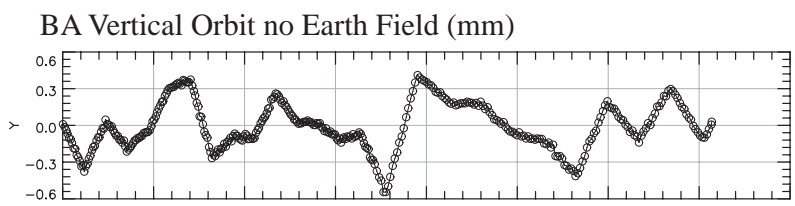

BA Vertical Orbit with Earth Field (mm)

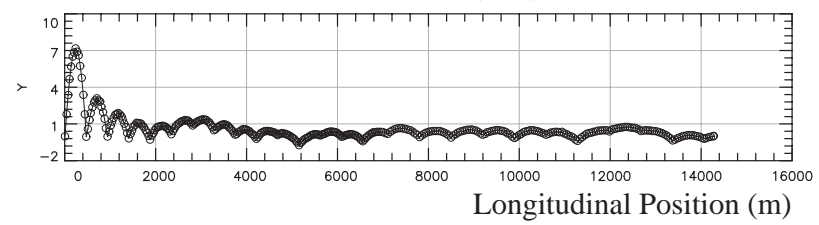

Figure 3: Beam orbit after ballistic alignment with and without Earth's magnetic field.

for all three alignment algorithms is shown in Figure 2. An emittance dilution budget of $100 \%(40 \mathrm{~nm})$ is also plotted as this reflects the current expectation [8]. This is twice the emittance budget used in previous analysis [6, 4]. Dispersion Free Steering uses differences between orbits for its analysis so there should be little or no sensitivity to a constant field as is illustrated in the figure. Ballistic Alignment assumes the beam undergoes a ballistic orbit when all linac components are turned off so an external field will have a dramatic effect on BA's ability to align the machine. Even a field of only $5 \mu \mathrm{T}$ is enough to increase the final emittance above the $100 \%$ budget. The Kubo method behaves in between these two extremes.

The majority of the emittance dilution due to Earth's field is when the beam has a relatively low energy near the beginning of the linac. Here, the beam is only tens of $\mathrm{GeV}$ in energy and it can be deflected by millimeters by the Earth's magnetic field. Figure 3 gives the beam orbit after ballistic alignment with and without Earth's magnetic field. If the shielding could be extended to fill the entire cryomodule for the first several hundred meters of the linac then the emittance dilution due to the millimeter-size deflected orbits could be removed. Figure 4 gives the final

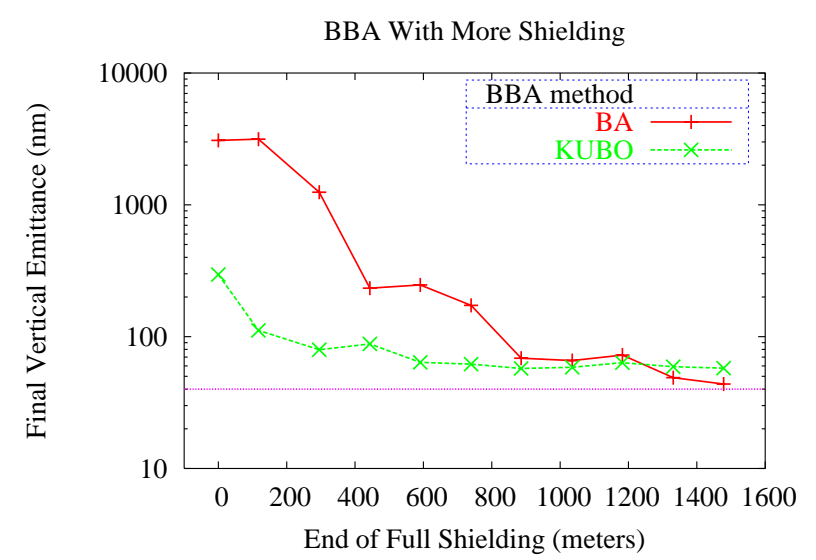

Figure 4: Final emittance dilution versus complete magnetic shielding. After the position listed, the magnetic shielding is only over the RF cavities.

emittance after BA and Kubo where the magnetic shielding is extended to fill the entire cryomodules up to the length given in the plot. For Ballistic Alignment, 1500 meters covers approximately the first three regions and is sufficient to reach the budget.

\section{CONCLUSIONS}

Ballistic Alignment and the Kubo method were found to agree well with previous calculations. Dispersion Free Steering was found to behave markedly worse when BPM noise is present. The source of the discrepancy is being investigated.

The Earth's magnetic field itself may not be a concern because it is predictable and compensation should be possible. However, the above analysis also shows the sensitivity of three beam-based alignment techniques to other unknown stray fields that may be present in the linac tunnel. If these fields are not measured then they could have a significant effect on Ballistic and Kubo alignment methods.

\section{REFERENCES}

[1] TESLA Technical Design Report, TESLA-Report 2001-13 (2001)

[2] D. Sagan, J. C. Smith, Proc. PAC 05 (2005)

[3] J. Urban, L. Fields, D. Sagan, Proc. PAC 05 (2005)

[4] D. Schulte, N. Walker, Proc. PAC 03 (2003)

[5] K. Kubo, March 2005, see: http://lcdev.kek.jp/ kkubo/reports/ MainLinac-simulation/lcsimu-20050325a.pdf

[6] P. Tenenbaum, R. Brinkmann, V. Tsakanov, Proc. EPAC 02 (2002)

[7] K. Ranjan, et al, April 2005, see: http://www-project.slac.stanford.edu/ilc/ acceldev/LET/talks.htm

[8] G. Dugan, private communication, March 2005 